\title{
Surgical.
}

\section{THE USE AND ABUSE OF PESSARIES.}

\author{
BY CHARLES D. READ, M.B., F.R.c.s., M.C.o.G. \\ Surgeon to Out-Patients, Chelsea Hospital for Women ; Obstctric and Gynacological Tutor, \\ Westminster Hospital.
}

THE great ingenuity of the earlier gynæcologists is adequately demonstrated by the many varied instruments devised for the alleviation of symptoms due to prolapse of the uterus and vaginal walls, and displacements of the uterus. Few of these instruments have survived the test of time, and with the great advance of plastic vaginal operative technique the indications for the use of pessaries have become limited. Indeed, years ago, the gynæcological out-patients' department of a hospital was litt'e more than a ringchanging clinic. With the passage of time, temporary methods of alleviation have given place to permanent methods of cure, and the modern out-patients' department harbours relatively few regular quarterly attenders for the purpose of changing pessaries. It seems safe to prophesy, however, that there will always be some place for the pessary in the armamentarium of the gynæcologist, and for these relatively few cases, three instruments - the rubber watch-spring pessary, the Hodge pessary and Napier's cup and stem pessary-will be found to fulfil all requirements.

At the best of times a pessary is a makeshift. It is surgically an unclean instrument, and its prolonged use is often attended by trauma to the vaginal walls and to the cervix uteri. In addition to these local changes prolonged use, in many cases, appears to lead to definite psychological trauma. This is especially so in the case of young women in whom the unavoidable mental concentration upon the pelvis appears to exert a deleterious effect, which produces the classical pelvic syndrome. Thanks to a more common-sense outlook, this is fast disappearing, but it must be admitted that even at the present time too many women are suffering the inconvenience of wearing instruments, little knowing what complete comfort operative treatment can offer. In addition, the operative cure can be effected with an almost negligible risk to life.

\section{The Rubber Watch-Spring Ring Pessary.}

Primarily this instrument was devised for the treatment of uterine prolapse, but recently its use has been extended to the treatment of retroversion of the gravid uterus, retroversion in association with prolapsed ovaries, and even to simple uterine retroversion. In addition, it sometimes serves a useful purpose in controlling incontinence of urine associated with prolapse, or even incontinence associated with a lax urethral sphincter in the absence of uterine or vaginal descent.

In the treatment of uterine prolapse its use is limited to descent of the first and second degrees. It is in extremely rare instances only that complete descent can be controlled by it, owing to an extremely deficient perineal body or a much relaxed vaginal 
introitus. In vault or virginal prolapse, when the uterus telescopes downwards into the vagina, it is often found that the smallness of the introitus will not allow of the insertion of an instrument sufficiently large to control the descent.

Broadly speaking, these are the indications for the use of this type of instrument, but even for these conditions, with the one exception of the retroverted gravid uterus, these indications are relative only, and should be limited to two types of patients : (a) Those who refuse to submit to operative treatment; $(b)$ those in whom an operative procedure would constitute a definite risk to life, such as severe cardiac disease, poor general condition, senility and diabetes.

The question of the young woman sufferer presents some difficulty. She is likely to have other children, and on this account many authorities advise temporary alleviation by pessary treatment until the age of 40 , when operative treatment is advised. This appears to be rather illogical. To condemn this patient to the wearing of an instrument at the time of life when she should be most active and fit, appears to be an unnecessary hardship when it is known that a correctly performed plastic vaginal operation will not only cure her, but will allow of her having further children without increased risk of recurrence of her trouble. This holds if the pregnancy and labour be efficiently managed. To my mind the youthfulness of the patient calls for early, rather than delayed operative cure.

\section{The Abuse of the Instrument.}

No instrument is more abused than the watch-spring pessary. It is used for als possible varieties of pelvic symptoms with little regard to the direct causes of these symptoms. The most frequent abuses seen by the gynæcologist are :-

(a) Use of the pessary for uncomplicated cases when the patient has not been offered the chance of operative cure. This surely is a gross misuse of the instrument.

(b) The blind use of the instrument for vague symptoms not in any way due to prolapse or displacement. The patient will not be relieved but will probably suffer more as a result, and a thorough pelvic examination will of ten reveal the true cause of the symptoms. If no pelvic lesion be found, the orthopædic surgeon will often be found helpful.

(c) Incorrect size of instrument used. The correct instrument is the largest that can be retained with the complete comfort of the patient. This can be well judged after insertion, by interposing the tip of the gloved index finger between the pessary and the vaginal mucosa. This done, without difficulty and without causing pain and in the presence of a close fit, indicates an accurately gauged instrument. If the size be incorrectly judged, discomfort will result. If too large, the over-stretching of the vaginal walls causes pain, and if too small the symptoms of the prolapse recur and, in addition, discomfort results from the presence of the ring low on the perineum.

(d) The use in cases of prolapse associated with vaginitis, cervicitis or salpingitis, is strongly deprecated. This leads to vaginal or cervical ulceration, or, in the case of salpingitis, to an acute exacerbation of the inflammatory process. The cervix and vagina must be treated by rest in bed, douches and the application of suitable antiseptics, until the inflammatory process has completely subsided. Then only may the instrument be introduced. In a similar manner the tubal disease must be eradicated by operation, and it will often be advisable to repair the prolapse at the same time. 
(e) Use of the instrument without adequate and proper instructions as to douching and changing the pessary. Daily douches with a non-irritant douche, such as Milton's, are necessary if the foreign body is to remain in the vagina without producing inflammatory changes. At intervals of three months the ring must be removed, the vagina swabbed out and douched and preferably a new instrument inserted. Rubber is a perishable commodity. Most gynæcologists have had the misfortune of having to remove a neglected ring pessary surrounded by ulceration and granulation tissue and occasionally associated with a fistula between either the bladder or the rectum and the vagina.

$(f)$ Misuse in cases of large cystocele or rectocele without uterine descent.

In the presence of a large cystocele or a rectocele without associated uterine descent the instrument is incapable of controlling the vaginal prolapse, as the anterior or the posterior vaginal wall descends at a much lower level than that controlled by the instrument. If the uterus descends as well, with these conditions, the general upward thrust maintained by the ring may serve to take up the vaginal walls, with alleviation of the symptoms.

\section{The Hodge Pessary.}

This instrument was originally designed for the maintenance of the position of anteversion of a uterus which has been replaced from the retroverted position. At the time of its invention it was believed that in almost every case retroversion was a serious and a symptom-producing displacement. In the light of recent knowledge it is agreed that, while a retroverted uterus may produce symptoms, it is often symptomless, and this is borne out by experience both in private and in hospital practice. With this in view, it would appear therefore that, as a general rule, it is necessary to treat these misplacements only in those cases which cause symptoms. In addition, the displacement must be a mobile one, i.e., one which allows of replacement. Everyone is agreed that fixed retroversions can be treated only by operative means. The uses of the Hodge instrument may be conveniently summarized as follows:-

(I) Temporary Use Attended by a Possibility of Permanent Cure.-(a) In traumatic retroversion, which is a rare displacement produced by a force or accident which causes a rise of intra-abdominal tension, in the presence of an over-distended bladder.

(b) In puerperal retroversion, diagnosed within a few weeks of miscarriage or delivery of a child at term. The maintenance of the forward position by the appliance allows of better uterine involution, and better involution of the over-stretched round ligaments and utero-sacral ligaments.

(2) Temporary Use in Uncomplicated Mobile Retroversion Associated with a History of Sterility or Repeated Miscarriages.-In both these cases the instrument is worn until pregnancy ensues, when it is advisable to replace the Hodge pessary by a rubber watchspring appliance which causes less pressure in the posterior fornix.

(3) Diagnostic Use.-This is a very useful and important means of ascertaining whether the symptoms complained of by a patient are due to the backward displacement present or otherwise. By replacement and insertion of a Hodge pessary as a diagnostic means, many unnecessary operations have been avoided. If the symptoms disappear, one concludes that the displacement is the cause, and the patient has the choice between pessary treatment and operation. The failure to alleviate the symptoms rules out the 
retroversion as the cause. If the symptoms are definitely exaggerated by the instrument one of the conditions mentioned later will be found.

(4) Use of the Modified Hodge Instrument for Cystocele.-This appliance is of the usual Hodge type, with a transverse vulcanite bar across its lower half. This serves to control descent of the anterior vaginal wall in the case of a large cystocele.

As in the case of the ring instrument, the Hodge pessary has a limited use. It is wise to give it a trial in all cases of recent traumatic and recent puerperal retroversion, and its use will be attended by many gratifying results. This also applies to its use in cases associated with sterility and repeated miscarriages. In some cases of sterility, however, its use appears to hinder conception, and if after a time conception does not result, it is justifiable to dilate the cervix and to insufflate the Fallopian tubes; if these are patent only when the uterus is anteverted, operative treatment of the displacement is indicated. In patients giving a history of repeated miscarriages, and in whom the position of the uterus cannot be adequately controlled by the instrument, operative treatment offers the best chance of pregnancy reaching term. The diagnostic use has been already discussed, and the treatment of cystocele by the modified appliance should be reserved for those patients who refuse operation or whose general condition contra-indicates operation because of the definite risk to life.

\section{Abuse of the Instrument.}

Like the watch-spring pessary, the instrument suffers many abuses from wrongfue use, with resulting suffering on the part of the patient. The commonest misuses are :

(I) Use in symptomless congenital retroversion in single women. Apart from the instrument's misuse, this constitutes a definite abuse of the patient.

(2) Use in old-standing symptomless displacements.

(3) Pain or discomfort following insertion always indicates something definitely amiss. The instrument should cause no discomfort whatever, and its presence in the vagina should not be appreciable to the patient. Pain always means one of the following contra-indications to the use of the instrument.

(a) Insertion without replacement of the uterus in the anterior position. The posterior bar then causes pressure upon the fundus and often upon the ovaries as well.

(b) Insertion in the presence of an acutely retroflexed uterus. This causes similar pressure. In rare instances retroflexion over the posterior bar occurs after previous anteversion of a recently subinvoluted uterus.

(c) The presence of prolapsed ovaries which are so markedly prolapsed that anteversion of the uterus fails to elevate them from the pouch of Douglas. Treatment in these cases is usually operative, especially if dyspareunia is a marked symptom. In some cases a ring pessary will relieve the patient.

(d) The treatment of a retroverted gravid uterus by a Hodge pessary is contraindicated because the pregnant uterus tends to retroflex over the posterior bar of the instrument and, in addition, the pressure exerted by the instrument may cause miscarriage. A ring pessary, which merely maintains the uterus in an upright position suffices.

(e) Faulty choice of size of instrument. If it is too large, pain results; if too small it is not retained. 
(g) The insertion of the instrument upside down. This is a very common error and may cause severe discomfort. The rounded end of the pessary is the posterior and upper extremity.

(h) Failure to guide the posterior bar over the cervix into the posterior vaginal fornix. On insertion, the upper and posterior bar almost invariably passes upwards into the anterior fornix, and it is always necessary to guide this over the cervix to the posterior fornix and, at the same time, to manipulate the lower and anterior bar under the pubic rami.

It can thus be seen that the use of this instrument is fraught with many pitfalls, all of which can be avoided by the observance of simple rules. If it is used appropriately, gratifying results are obtained. The patient wearing the instrument should douche her vagina on alternate days with a non-irritating solution, and at intervals of three months the appliance should be removed and cleaned, and the vagina should be douched before replacement of the pessary, if this is necessary.

\section{Napier's Cup and Stem Pessary.}

In general, the use of this instrument must be regarded as a last resort. Its use is restricted to cases of procidentia in which both operative and ring pessary treatment are impossible; the former because of some associated constitutional condition contraindicating operation, or because of refusal to submit to operation, and the latter because of inability to retain a ring due to marked deficiency of the perineal body or laxity of the vaginal introitus. Under similar conditions, second degree prolapse may require this treatment.

The vagina and cervix must be healthy, and if this is not so, treatment must be instituted to attain this. The cup must be of sufficient dimensions to control the hernial orifice of the prolapse, and the stem must be sufficiently long to maintain the uterus and the vaginal walls at their correct level.

In conclusion, from what has been stated, it can be seen that pessaries have a definite though limited use in gynæcological practice. The abuses of the instruments concerned outnumber the uses to a considerable extent, but the abuses are not the fault of the instruments but of the people who misuse them. The analogy of the bad workman and his tools, holds. The limitation of the use of instruments for uterine prolapse and displacements is mainly due to the increased efficiency and the decreased risk of vaginal plastic surgery. 